ECCOMAS Proceedia
UNCECOMP 2021

$4^{\text {th }}$ ECCOMAS Thematic Conference on Uncertainty Quantification in Computational Sciences and Engineering M. Papadrakakis, V. Papadopoulos, G. Stefanou (eds.) Streamed from Athens, Greece, 28 -30 June 2021

\title{
FEM SHAKEDOWN ANALYSIS OF KIRCHHOFF-LOVE PLATES UNDER UNCERTAINTY OF STRENGTH
}

\author{
Ngọc Trình Trần ${ }^{1}$ and Manfred Staat ${ }^{2}$ \\ ${ }^{1}$ Hanoi Architectural University \\ Hanoi, Vietnam \\ e-mail: trinhdhkt@gmail.com \\ ${ }^{2}$ FH Aachen University of Applied Sciences, Germany \\ Institute of Bioengineering \\ Heinrich-Mußmann-Str. 1, 52428 Jülich, Germany \\ e-mail:m.staat@fh-aachen.de
}

\begin{abstract}
A new formulation to calculate the shakedown limit load of Kirchhoff plates under stochastic conditions of strength is developed. Direct structural reliability design by chance constrained programming is based on the prescribed failure probabilities, which is an effective approach of stochastic programming if it can be formulated as an equivalent deterministic optimization problem.

We restrict uncertainty to strength, the loading is still deterministic. A new formulation is derived in case of random strength with lognormal distribution. Upper bound and lower bound shakedown load factors are calculated simultaneously by a dual algorithm.
\end{abstract}

Keywords: Kirchhoff Plate, Limit Analysis, Shakedown Analysis, Primal Dual Programming, Stochastic Programming, Chance Constrained Programming. 


\section{INTRODUCTION}

Plates are very important structural elements, which are widely used in civil and mechanical engineering. The common examples of plates are slabs in civil engineering structures, bearing plate under columns, many parts of mechanical components. In this chapter, we consider bending of such plates subjected to lateral loads. The bending stiffness of a plate depends on the cube of its thickness. The classical theory divides plates into following groups: thin plates with small deflection, thin plates with large deflections, and thick plates.

The following assumptions are made in the small deflections theory of thin plates:

a) There is no deformation in the middle plane of the plate. This plane remains neutral during bending.

b) The normal to the middle plane of the plate remains straight and normal to the deformed middle plane.

c) The normal stresses in the transverse direction to the plate are negligible.

The above assumptions on which A.E.H. Love based his plate theory were proposed by Gustav R. Kirchhoff [1]. Consequently, thin plates with small deflections theory are called Kirchhoff-Love plate or Kirchhoff plate for short. This theory is suitable for plates with length of span at least 10 times the thickness. Many engineering problems lie in the above category and satisfactory results are obtained by the classical thin plates theory.

If the span is less than 10 times the thickness, the thin plates assumptions (a) and (b) no longer apply. The Reissner-Mindlin plate thick plates theory, which accounts for shear deformations, or a three dimensional analysis can be recommended for such plates [12].

Limit analysis of plates in bending has been studied analytically and numerically [11]-[23]. Due to limitations of analytical methods, alternative numerical approaches such as finite element methods (FEM), meshfree methods or isogeometric analysis (IGA) have been developed.

In [24] a dual algorithm has been developed to calculate simultaneously both the upper and lower bounds of the plastic collapse limit and shakedown limit of thin plates. We reformulate a similar algorithm as deterministic equivalent of a chance constrained program in which the lower bound and upper bound limit and shakedown load of plate under uncertain strength is computed.

Limit and shakedown analysis state problems as a mathematical programming. If the strength of a plate is a random variable, we may consider the problems as a stochastic programming problem. Many models of stochastic programming have been proposed such as approximate polyhedral dynamic programming [25]-[27], nominal solutions [28], measurementbased optimization [29], [30], worst-case and distributional robustness analysis [31]-[33], robust optimization [34], recourse programming [35]-[37] and chance constrained optimization (CCOPT) [38], [39]. In this paper the CCOPT approach is used to treat the problem of shakedown analysis of plate under uncertainty condition of strength. If the thickness deterministic and the yield stress is distributed normally or lognormaly a deterministic equivalent formulation can be derived, which allows a most effective numerical calculation of limit and shakedown loads for a prescribed failure probability of the structure.

\section{BASIC RELATIONS IN THIN PLATE THEORY}

In this section the necessary relations are listed using the notations as indicated in the plate element shown in Fig. 1. 


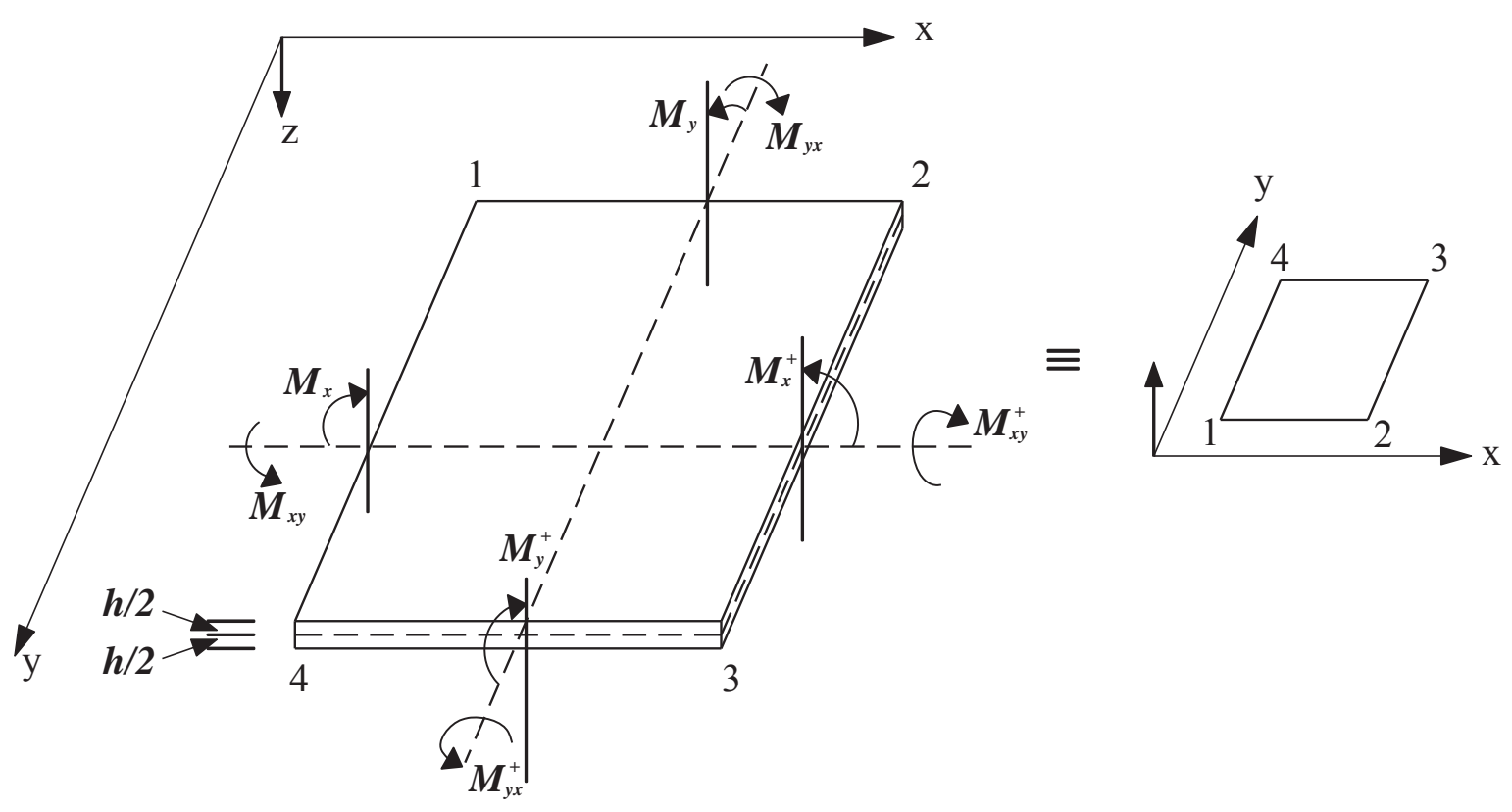

Figure 1: Plate element with internal moment resultants

Let $u, v$ and $w$ be the displacement at any point $(x, y, z)$ in the plate. Similar to elasticity theory, the inelastic behavior of thin plates is analyzed under Kirchhoff's assumption that the normal to the middle plane of the plate remains straight and normal to the deformed middle plane. This assumption yields $u=-z \frac{\partial w}{\partial x}, v=-z \frac{\partial w}{\partial y}$ and the strains are obtained as

$$
\left\{\begin{array}{c}
\varepsilon_{x x} \\
\varepsilon_{y y} \\
\gamma_{x y}
\end{array}\right\}=\left\{\begin{array}{c}
\frac{\partial u}{\partial x} \\
\frac{\partial v}{\partial y} \\
\frac{\partial v}{\partial x}+\frac{\partial u}{\partial y}
\end{array}\right\}=\left\{\begin{array}{c}
-z \frac{\partial^{2} w}{\partial x^{2}} \\
-z \frac{\partial^{2} w}{\partial y^{2}} \\
-2 z \frac{\partial^{2} w}{\partial x \partial y}
\end{array}\right\}=z\left\{\begin{array}{c}
\chi_{x} \\
\chi_{y} \\
\chi_{x y}
\end{array}\right\} .
$$

Here $\{\chi\}=\left[\begin{array}{lll}\chi_{x} & \chi_{y} & \chi_{x y}\end{array}\right]^{T}$ is the vector of curvatures. The kinematic relations can be written as follows:

$$
\dot{\chi}=-\nabla^{2} \dot{w},
$$

where $\dot{\chi}$ is the curvature rate vector and $\dot{w}$ is the transversal velocity.

\subsection{Yield criteria}

Similar to fully plastic beams, the limit state of greatest load carrying capacity is obtained for a double rectangular distribution of the stresses across the thickness $h$ of the plate. Therefore, the limit values of the bending moments in the $x$ and $y$ directions and of the twisting moment are

$$
M_{x}=\sigma_{x} \frac{h^{2}}{4}, M_{y}=\sigma_{y} \frac{h^{2}}{4}, M_{x y}=\sigma_{x y} \frac{h^{2}}{4}
$$


Contrary to the bending of beams, however, the normal stresses $\sigma_{x}$ and $\sigma_{y}$ are not equal to the yield limits in uniaxial tension; rather, they must satisfy a yield condition for plane stress, taking into account the in-plane shear stress $\sigma_{x y}$. The out-of-plane shear stresses, $\sigma_{x z}, \sigma_{y z}$ are usually neglected. Consider a general yield condition of the form

$$
f\left(\sigma_{x}, \sigma_{y}, \sigma_{x y}\right)=0
$$

applicable to plane stress states. In a fully plasticized cross section, the stresses $\sigma_{x}, \sigma_{y}, \sigma_{x y}$ are constant. Expressing (2.4) in terms of bending and twisting moments, we have the corresponding yield criterion for a plate,

$$
f\left(\frac{4 M_{x}}{h^{2}}, \frac{4 M_{y}}{h^{2}}, \frac{4 M_{x y}}{h^{2}}\right)=0 .
$$

As an illustration, the von Mises yield criterion can be written in the form

$$
f\left(\sigma_{x}, \sigma_{y}, \sigma_{x y}\right) \equiv \sigma_{x}^{2}-\sigma_{x} \sigma_{y}+\sigma_{y}^{2}+3 \sigma_{x y}^{2}-\sigma_{0}^{2}=0 .
$$

Expressing from (2.6) stresses in terms of moments, the criterion takes the form

$$
f\left(M_{x}, M_{y}, M_{x y}\right) \equiv M_{x}^{2}-M_{x} M_{y}+M_{y}^{2}+3 M_{x y}^{2}-M_{0}^{2}=0
$$

in which

$$
M_{0}=\sigma_{0} \frac{h^{2}}{4} .
$$

In matrix form, the von Mises yield criterion can be written as follows:

$$
f(\mathbf{m})=\sqrt{\mathbf{m}^{\mathrm{T}} \mathbf{P m}}-m_{0}=0,
$$

where $\mathbf{m}=\left\{M_{x}, M_{y}, M_{x y}\right\}^{\mathrm{T}}$ is the vector of bending and twisting moments, $m_{0} \equiv M_{0}$ is the fully plastic limit moment per unit length of a plate section and $\sigma_{0}$ is the uniaxial yield stress of material,

$$
\mathbf{P}=\frac{1}{2}\left[\begin{array}{ccc}
2 & -1 & 0 \\
-1 & 2 & 0 \\
0 & 0 & 6
\end{array}\right\rfloor
$$

A more complex yield surface of plates and shells has been considered in [40].

\section{STATIC APPROACH WITH CHANCE CONSTRAINED PROGRAMMING}

Consider a convex polyhedral load domain $\mathcal{L}$ and a special loading path consisting of all load vertices $\hat{P}_{k}(k=1, \ldots, m)$ of $\mathcal{L}$. The total moment $\mathbf{m}(\mathbf{x}, t)$ at a point $\mathbf{x} \in \Omega$ of the considered plate $\mathscr{P}$ at time $t$ is decomposed into an elastic reference moment $\mathbf{m}^{E}(\mathbf{x}, t)$ and a residual moment $\boldsymbol{\rho}(\mathbf{x}, t)$. Here, $\mathbf{m}^{E}(\mathbf{x}, t)$ denotes the fictitious moment that would appear in a purely elastic reference structure $\mathscr{P}^{E}$ under the same loading conditions as the original struc- 
ture, and $\boldsymbol{\rho}(\mathbf{x}, t)$ represents a residual moment field that is induced by the evolution of plastic strains

$$
\mathbf{m}(\mathbf{x}, t)=\mathbf{m}^{E}(\mathbf{x}, t)+\boldsymbol{\rho}(\mathbf{x}, t) .
$$

According to Melan's static shakedown theorem the structure will shakedown, if there exists a time-independent residual moment field $\overline{\boldsymbol{\rho}}(\mathbf{x})$ such that the yield condition is satisfied for any loading path at any time $t$ and in any point $\mathbf{x}$ of the plate. Based on this lower bound theorem, for a plate made up of elastic perfectly plastic material, the maximum enlarging of the load domain allowing still for shakedown, characterized by load factor $\alpha^{-}$that can be obtained by solving the following optimization problem:

$$
\begin{aligned}
& \alpha^{-}=\max \alpha \\
& \text { s.t.: }\left\{\begin{array}{l}
\nabla^{2} \overline{\boldsymbol{\rho}}(\mathbf{x})=0 \quad \text { in } \Omega \\
f\left[\alpha \mathbf{m}^{\mathrm{E}}(\mathbf{x}, t)+\overline{\boldsymbol{\rho}}(\mathbf{x})\right] \leq m_{0}
\end{array}\right.
\end{aligned}
$$

By discretizing the entire problem domain $\Omega$ into finite elements and applying the GaussLegendre integration technique, eqs. (3.2) can be rewritten in the following form:

$$
\begin{aligned}
& \alpha^{-}=\max \alpha \\
& \text { s.t.: }\left\{\begin{array}{l}
\sum_{i=1}^{N G} w_{i} \mathbf{B}_{i}^{T} \overline{\boldsymbol{\rho}}_{i}=0 \quad \text { in } \Omega \\
f\left(\alpha \mathbf{m}_{i k}^{E}+\overline{\boldsymbol{\rho}}_{i}\right) \leq m_{0} \quad \forall i=\overline{1, N G} \quad \forall k=\overline{1, m}
\end{array}\right.
\end{aligned}
$$

in which $\mathbf{B}_{i}$ is the deformation matrix, $w_{i}$ is integration weight at Gauss point $i$ and $N G$ denotes the total number of Gauss points of the structure.

Let us now consider the situation that the plastic moment of the plate is not given but must be modelled $m_{0}=m_{0}(\omega)$ a random variable on a certain probability space. Under uncertainty, the inequalities in (3.3) are not always satisfied, the probability of the $i^{\text {th }}$ yield condition is required to be satisfied is greater than some reliability level $\psi_{i}$. Problem (3.3) becomes a chance constraint stochastic program:

$$
\begin{aligned}
& \alpha^{-}=\max \alpha \\
& \text { s.t.: }\left\{\begin{array}{l}
\sum_{i=1}^{N G} w_{i} \mathbf{B}_{i}^{T} \overline{\boldsymbol{\rho}}_{i}=\mathbf{0} \quad \text { in } \Omega \\
\operatorname{Prob}\left[f\left(\alpha \mathbf{m}_{i k}^{E}+\overline{\boldsymbol{\rho}}_{i}\right)-m_{0 i}(\omega) \leq 0\right] \geq \psi_{i} \quad \forall i=\overline{1, N G} \quad \forall k=\overline{1, m}
\end{array}\right.
\end{aligned}
$$

Let the plastic moment $m_{i}(\omega)$ be distributed normally with mean $\mu_{i}$ and standard deviation $\sigma_{i}$, in short $m_{i}(\omega) \sim \mathcal{N}\left(\mu_{i}, \sigma_{i}^{2}\right)$. Based on the methodology of chance constrained programming, problem (3.4) can be converted into a equivalent deterministic program as shown in [10], [11]: 


$$
\begin{aligned}
& \alpha^{-}=\max \alpha \\
& \text { s.t.: }\left\{\begin{array}{l}
\sum_{i=1}^{N G} w_{i} \mathbf{B}_{i}^{T} \overline{\boldsymbol{\rho}}_{i}=0 \quad \text { in } \Omega \\
f\left(\alpha \mathbf{m}_{i k}^{E}+\overline{\boldsymbol{\rho}}_{i}\right) \leq \mu_{i}-\kappa \sigma_{i} \quad \forall i=\overline{1, N G} \quad \forall k=\overline{1, m}
\end{array}\right.
\end{aligned}
$$

where $\kappa=\Phi^{-1}\left(\psi_{i}\right)$ is the inverse normal cumulative distribution function (normal quantile function) of the plastic moment at Gauss point $i$.

Let the plastic moment $m_{i}(\omega)$ be distributed lognormally. This means that $\ln \left[m_{i}(\omega)\right]$ is distributed normally with mean $\mu_{i}$ and standard deviation $\sigma_{i}$, in short $\ln \left[m_{i}(\omega)\right] \sim \mathcal{N}\left(\mu_{i}, \sigma_{i}^{2}\right)$. The stochastic program (3.4) can be relaxed into an equivalent deterministic optimization problem after some transformations [2,3]:

$$
\begin{aligned}
& \alpha^{-}=\max \alpha \\
& \text { s.t.: }\left\{\begin{array}{l}
\sum_{i=1}^{N G} w_{i} \mathbf{B}_{i}^{T} \overline{\mathbf{\rho}}_{i}=0 \quad \text { in } \Omega \\
f\left(\alpha \mathbf{m}_{i k}^{E}+\overline{\boldsymbol{\rho}}_{i}\right) \leq e^{\mu_{i}-\kappa \sigma_{i}} \quad \forall i=\overline{1, N G} \quad \forall k=\overline{1, m}
\end{array}\right.
\end{aligned}
$$

\section{KINEMATIC APPROACH WITH CHANCE CONSTRAINED PROGRAMMING}

An upper bound to the shakedown limit of plates can be obtained using the kinematic shakedown theorem which has following two statements:

Upper bound: Shakedown will occur for a structure subject to repeated or cyclic loads, if the rate of plastic dissipation power exceeds the work rate of external forces for any admissible plastic strain-rate cycles and all loading paths.

Lower bound: Shakedown cannot occur, if the rate of plastic dissipation power is less than the work rate of external forces for any one admissible plastic strain-rate cycle or any one loading path.

In this investigation, we use von Mises yield criterion. The power of plastic dissipation per unit area of the plate can be formulated as a function of strain rate:

$$
\dot{D}_{p}=\sigma_{0} \sqrt{\dot{\boldsymbol{\varepsilon}}^{T} \mathbf{Q} \dot{\boldsymbol{\varepsilon}}}
$$

where (with (2.10))

$$
\mathbf{Q}=\mathbf{P}^{-1}=\frac{1}{3}\left[\begin{array}{lll}
4 & 2 & 0 \\
2 & 4 & 0 \\
0 & 0 & 1
\end{array}\right\rfloor
$$

The plastic dissipation power of the plate domain $\Omega$ can be written

$$
\dot{D}_{\text {int }}(\dot{\chi})=\int_{\Omega} \int_{-h / 2}^{h / 2} \dot{D}_{p} \mathrm{~d} z \mathrm{~d} \Omega=m_{0} \int_{\Omega} \sqrt{\dot{\chi}^{T} \mathbf{Q} \dot{\chi}} \mathrm{d} \Omega
$$

in which $m_{0}$ is the plastic limit moment per unit length of a plate section is computed as (2.8) 
We introduce here an admissible cycle of a plastic curvature field $\Delta \chi^{p}$. At each load vertex, the plastic curvature rate may not necessarily be compatible at each instant during the time cycle, but the plastic curvature accumulation over the cycle is required to be kinematically compatible such that

$$
\Delta \chi^{p}=\sum_{k=1}^{m} \dot{\chi}^{p}=\nabla^{2} \dot{w}
$$

Based on the above statements and the mathematical programming theory, an upper bound of the shakedown load factor can be found by solving the following convex nonlinear programming (the superscript $p$ is neglected for simplicity):

$$
\begin{aligned}
& \alpha^{+}=\min \sum_{k=1}^{m} \int_{A} \dot{D}_{\text {int }}(\dot{\chi}) \mathrm{d} \Omega \\
& \text { s.t.: }\left\{\begin{array}{l}
\Delta \chi^{p}=\sum_{k=1}^{m} \dot{\chi}=\nabla^{2} \dot{w} \quad \text { in } \Omega \\
\dot{w}=0 \quad \text { on } \partial \Omega \\
\sum_{k=1}^{m} \int_{\Omega} \mathbf{m}^{E}\left(x, \hat{P}_{k}\right) \dot{\chi}^{T} \mathrm{~d} \Omega=1
\end{array}\right.
\end{aligned}
$$

We denote the nodal variables of the finite element by $\mathbf{u}=\left[\begin{array}{lll}w & \partial w / \partial x & \partial w / \partial y\end{array}\right]^{\mathrm{T}}$. The discretized formulation by FEM is as follows:

$$
\begin{aligned}
& \alpha^{+}=\min \sum_{k=1}^{m} \sum_{i=1}^{N G} w_{i} m_{0} \sqrt{\dot{\boldsymbol{\chi}}_{i k}^{\mathrm{T}} \mathbf{Q} \dot{\boldsymbol{\chi}}_{i k}} \\
& \text { s.t.: }\left\{\begin{array}{l}
\sum_{k=1}^{m} \dot{\boldsymbol{\chi}}_{i k}=\mathbf{B}_{i} \dot{\mathbf{u}} \quad \forall i=\overline{1, N G} \\
\sum_{k=1}^{m} \sum_{i=1}^{N G} w_{i} \dot{\boldsymbol{\chi}}_{i k}^{\mathrm{T}} \mathbf{m}_{i k}^{E}=1
\end{array}\right.
\end{aligned}
$$

If the yield stress of the material is random, then the plastic moment is an uncertain quantity and the objective function of (4.6) is a stochastic variable. Firstly, we must properly define the minimum of a random function. This can be done in such a way that one looks for a minimum lower bound $\eta$ of the objective function under the constraint that the probability of violation of that bound is prescribed in [39]

$$
\text { s.t.: }\left\{\begin{array}{l}
\operatorname{Prob}\left(\sum_{k=1}^{m} \sum_{i=1}^{N G} w_{i} m_{0}(\omega) \sqrt{\dot{\boldsymbol{\chi}}_{i k}^{\mathrm{T}} \mathbf{Q} \dot{\boldsymbol{\chi}}_{i k}} \geq \eta\right)=\psi \\
\sum_{k=1}^{m} \dot{\boldsymbol{\chi}}_{i k}=\mathbf{B}_{i} \dot{\mathbf{u}} \quad \forall i=\overline{1, N G} \\
\sum_{k=1}^{m} \sum_{i=1}^{N G} w_{i} \dot{\boldsymbol{\chi}}_{i k}^{\mathrm{T}} \mathbf{m}_{i k}^{E}=1
\end{array}\right.
$$

Problem (4.7) is a stochastic program, which can be converted into an equivalent deterministic program by using a chance constrained programming technique [10], [11]. 


$$
\begin{array}{r}
\alpha^{+}=\min \sum_{k=1}^{m} \sum_{i=1}^{N G} w_{i}\left(\mu_{i}-\kappa \sigma_{i}\right) \sqrt{\dot{\boldsymbol{\chi}}_{i k}^{\mathrm{T}} \mathbf{Q} \dot{\chi}_{i k}} \\
\text { s.t.: }\left\{\begin{array}{l}
\sum_{k=1}^{m} \dot{\chi}_{i k}=\mathbf{B}_{i} \dot{\mathbf{u}} \quad \forall i=\overline{1, N G} \\
\sum_{k=1}^{m} \sum_{i=1}^{N G} w_{i} \dot{\boldsymbol{\chi}}_{i k}^{\mathrm{T}} \mathbf{m}_{i k}^{E}=1
\end{array}\right.
\end{array}
$$

In case of a lognormal distribution of strength, the stochastic problem (4.7) can be converted into the equivalent deterministic program (4.9) by using the duality property [3]:

$$
\begin{array}{r}
\alpha^{+}=\min \sum_{k=1}^{m} \sum_{i=1}^{N G} w_{i} e^{\left(\mu_{i}-\kappa \sigma_{i}\right)} \sqrt{\dot{\boldsymbol{\chi}}_{i k}^{\mathrm{T}} \mathbf{Q} \dot{\chi}_{i k}} \\
\text { s.t. }:\left\{\begin{array}{l}
\sum_{k=1}^{m} \dot{\chi}_{i k}=\mathbf{B}_{i} \dot{\mathbf{u}} \quad \forall i=\overline{1, N G} \\
\sum_{k=1}^{m} \sum_{i=1}^{N G} w_{i} \dot{\chi}_{i k}^{\mathrm{T}} \mathbf{m}_{i k}^{E}=1
\end{array}\right.
\end{array}
$$

\section{A DUAL ALGORITHM FOR SHAKEDOWN ANALYSIS OF A KIRCHHOFF PLATE}

For the sake of simplicity, we set some new notations:

$$
\dot{\mathbf{k}}_{i k}=w_{i} \mathbf{Q}^{1 / 2} \dot{\boldsymbol{\chi}}_{i k}, \quad \mathbf{t}_{i k}=\left(\mathbf{Q}^{-1 / 2}\right)^{T} \mathbf{m}_{i k}^{E}, \quad \hat{\mathbf{B}}_{i}=w_{i} \mathbf{Q}^{1 / 2} \mathbf{B}_{i},
$$

where

$$
\mathbf{Q}^{1 / 2} \mathbf{Q}^{-1 / 2}=\mathbf{I}, \quad \mathbf{Q}=\left(\mathbf{Q}^{1 / 2}\right)^{T} \mathbf{Q}^{1 / 2}
$$

By substituting (5.1) into (4.10) one obtains a simplified version for the upper bound of the shakedown limit load (primal problem)

$$
\begin{aligned}
& \alpha^{+}=\min \sum_{k=1}^{m} \sum_{i=1}^{N G} e^{\left(\mu_{i}-\kappa \sigma_{i}\right)} \sqrt{\dot{\mathbf{k}}_{i k}^{T} \dot{\mathbf{k}}_{i k}} \\
& \text { s.t. }: \begin{cases}\sum_{k=1}^{m} \dot{\mathbf{k}}_{i k}-\hat{\mathbf{B}}_{i} \dot{\mathbf{u}}=\mathbf{0} & \forall i=\overline{1, N G} \\
\sum_{i=1}^{N G} \sum_{k=1}^{m} \dot{\mathbf{k}}_{i k}^{T} \mathbf{t}_{i k}-1=0 & \end{cases}
\end{aligned}
$$

In order to allow a direct nonlinear of the nonsmooth optimization problem, a 'smooth regularization method' can be used for overcoming this technical problem. For this purpose, a very small positive number $\varepsilon_{0}^{2}$ is added to $D_{\text {int }}\left(\dot{\mathbf{k}}_{i k}\right)$. An efficient technique for large-scale optimization problems, which are successfully applied in [9] is used. Using a penalty method to eliminate the first constraint in (5.3) leads to the penalty function

$$
F_{p}=\sum_{i=1}^{N G}\left\{\sum_{k=1}^{m} e^{\left(\mu_{i}-\kappa \sigma_{i}\right)} \sqrt{\dot{\mathbf{k}}_{i k}^{T} \dot{\mathbf{k}}_{i k}+\varepsilon_{0}^{2}}+\frac{c}{2}\left(\sum_{k=1}^{m} \dot{\mathbf{k}}_{i k}-\hat{\mathbf{B}}_{i} \dot{\mathbf{u}}\right)^{T}\left(\sum_{k=1}^{m} \dot{\mathbf{k}}_{i k}-\hat{\mathbf{B}}_{i} \dot{\mathbf{u}}\right)\right\},
$$


where $c$ is a penalty parameter such that $c \gg 1$. The corresponding Lagrange function of (5.4) is

$$
L=F_{p}-\alpha\left(\sum_{i=1}^{N G} \sum_{k=1}^{m} \dot{\mathbf{k}}_{i k}^{T} \mathbf{t}_{i k}-1\right)
$$

We denote

$$
\boldsymbol{\beta}_{i}=-C\left(\sum_{k=1}^{m} \dot{\mathbf{k}}_{i k}-\hat{\mathbf{B}}_{i} \dot{\mathbf{u}}\right)
$$

By employing Newton method to solve the Karush-Kuhn-Tucker (KKT) conditions of the Lagrange function (4.17) and after some manipulations, one gets the following system:

$$
\mathbf{K d} \dot{\mathbf{u}}=-\mathbf{K} \dot{\mathbf{u}}+\mathbf{f}_{1}+\mathbf{f}_{2}(\alpha+\mathrm{d} \alpha),
$$

in which

$$
\begin{aligned}
& \mathbf{K}=\sum_{i=1}^{N G} \hat{\mathbf{B}}_{i}^{T} \mathbf{E}_{i}^{-1} \hat{\mathbf{B}}_{i} \\
& \mathbf{f}_{1}=-\sum_{i=1}^{N G} \hat{\mathbf{B}}_{i}^{T} \mathbf{E}_{i}^{-1} \sum_{k=1}^{m} \mathbf{M}_{i k}^{-1}\left(\boldsymbol{\beta}_{i}+\alpha \mathbf{t}_{i k}\right) \frac{\dot{\mathbf{k}}_{i k}^{T} \dot{\mathbf{k}}_{i k}}{\sqrt{\dot{\mathbf{k}}_{i k}^{T} \dot{\mathbf{k}}_{i k}+\varepsilon_{0}^{2}}} \\
& \mathbf{f}_{2}=\sum_{i=1}^{N G} \hat{\mathbf{B}}_{i}^{T} \mathbf{E}_{i}^{-1} \sum_{k}^{m} \mathbf{M}_{i k}^{-1} \sqrt{\dot{\mathbf{k}}_{i k}^{T} \dot{\mathbf{k}}_{i k}+\varepsilon_{0}^{2}} \mathbf{t}_{i k}
\end{aligned}
$$

and

$$
\begin{aligned}
& \mathbf{M}_{i k}=e^{\left(\mu_{i}-k \sigma_{i}\right)} \mathbf{I}+\left(\boldsymbol{\beta}_{i}+\alpha \mathbf{t}_{i k}\right) \frac{\dot{\mathbf{k}}_{i k}^{T}}{\sqrt{\dot{\mathbf{k}}_{i k}^{T} \dot{\mathbf{k}}_{i k}+\varepsilon_{0}^{2}}} \\
& \mathbf{E}_{i}=\frac{\mathbf{I}}{C}+\sum_{k}^{m} \mathbf{M}_{i k}^{-1} \sqrt{\dot{\mathbf{k}}_{i k}^{T} \dot{\mathbf{k}}_{i k}+\varepsilon_{0}^{2}}
\end{aligned}
$$

The system (4.19) with the two last terms on the right-hand side may be interpreted as the linear system arising in purely elastic computations with the global stiffness matrix $\mathbf{K}$. The matrix $\mathbf{E}_{i}^{-1}$ plays the role of the elastic matrix. Solving this system by the same procedure as for the purely elastic calculation will ensure the kinematic boundary condition for the displacement rate to be satisfied automatically. We have the incremental vectors of nodal variables $\dot{\mathbf{u}}$, curvature rate $\dot{\mathbf{k}}_{i k}$ and $\boldsymbol{\beta}_{i}$ as follows :

$$
\begin{aligned}
& d \dot{\mathbf{u}}=d \dot{\mathbf{u}}_{1}+d \dot{\mathbf{u}}_{2}(\alpha+d \alpha) \\
& d \dot{\mathbf{k}}_{i k}=\left(d \dot{\mathbf{k}}_{i k}\right)_{1}+\left(d \dot{\mathbf{k}}_{i k}\right)_{2}(\alpha+d \alpha) \\
& d \boldsymbol{\beta}_{i}=\left(d \boldsymbol{\beta}_{i}\right)_{1}+\left(d \boldsymbol{\beta}_{i}\right)_{2}(\alpha+d \alpha)
\end{aligned}
$$

where 


$$
\begin{aligned}
& d \dot{\mathbf{u}}_{1}=-\dot{\mathbf{u}}+\mathbf{K}^{-1} \mathbf{f}_{1} \\
& d \dot{\mathbf{u}}_{2}=\mathbf{K}^{-1} \mathbf{f}_{2} \\
& \left(d \dot{\mathbf{k}}_{i k}\right)_{1}=-\mathbf{M}_{i k}^{-1} \sqrt{\dot{\mathbf{k}}_{i k}^{T} \dot{\mathbf{k}}_{i k}+\varepsilon_{0}^{2}}\left(d \boldsymbol{\beta}_{i}\right)_{1}-\mathbf{M}_{i k}^{-1}\left(\bar{m}_{i} \dot{\mathbf{k}}_{i k}+\sqrt{\dot{\mathbf{k}}_{i k}^{T} \dot{\mathbf{k}}_{i k}+\varepsilon_{0}^{2}} \boldsymbol{\beta}_{i}\right) \\
& \left(d \dot{\mathbf{k}}_{i k}\right)_{2}=-\mathbf{M}_{i k}^{-1} \sqrt{\dot{\mathbf{k}}_{i k}^{T} \dot{\mathbf{k}}_{i k}+\varepsilon_{0}^{2}}\left(d \boldsymbol{\beta}_{i}\right)_{2}-\mathbf{M}_{i k}^{-1} \sqrt{\dot{\mathbf{k}}_{i k}^{T} \dot{\mathbf{k}}_{i k}+\varepsilon_{0}^{2}} \mathbf{t}_{i k} \\
& \left(d \boldsymbol{\beta}_{i}\right)_{1}=-\mathbf{E}_{i}^{-1} \sum_{k}^{m} \mathbf{M}_{i k}^{-1} \bar{m}_{i} \dot{\mathbf{k}}_{i k}-\mathbf{E}_{i}^{-1}\left[\hat{\mathbf{B}}_{i} d \dot{\mathbf{u}}_{1}-\left(\sum_{k=1}^{m} \dot{\mathbf{k}}_{i k}-\hat{\mathbf{B}}_{i} \dot{\mathbf{u}}\right)\right]-\boldsymbol{\beta}_{i} \\
& \left(d \boldsymbol{\beta}_{i}\right)_{2}=-\mathbf{E}_{i}^{-1} \hat{\mathbf{B}}_{i} d \dot{\mathbf{u}}_{2}-\mathbf{E}_{i}^{-1} \sum_{k}^{m} \mathbf{M}_{i k}^{-1} \sqrt{\dot{\mathbf{k}}_{i k}^{T} \dot{\mathbf{k}}_{i k}+\varepsilon_{0}^{2}} \mathbf{t}_{i k}
\end{aligned}
$$

and

$$
(\alpha+d \alpha)=\frac{1-\sum_{i=1}^{N G} \sum_{k=1}^{m} \mathbf{t}_{i k}^{T}\left[\dot{\mathbf{k}}_{i k}+\left(d \dot{\mathbf{k}}_{i k}\right)_{1}\right]}{\sum_{i=1}^{N G} \sum_{k=1}^{m} \mathbf{t}_{i k}^{T}\left(d \dot{\mathbf{k}}_{i k}\right)_{2}}
$$

The vectors d $\dot{\mathbf{q}}, \mathrm{d} \dot{\mathbf{k}}_{i k}, \mathrm{~d} \boldsymbol{\beta}_{i}$ and $\mathrm{d} \alpha$ are actually Newton directions, which assure that a suitable step along them will lead to a decrease of the objective function of the primal problem (5.3) and to an increase of the objective function of the objective function of the dual problem (3.8). Based on (5.9-5.11) we can update the vectors of $\dot{\mathbf{q}}, \dot{\mathbf{k}}_{i k}, \boldsymbol{\beta}_{i}$ and $\alpha$. The dual algorithm for limit and shakedown analysis is presented in detail in [3].

\section{NUMERICAL EXAMPLES}

We investigate a L-shape plate subjected to uniform pressure. Length $L=10 \mathrm{~m}$, plate thickness $t=0.1 \mathrm{~m}$, the mean value of yield stress $E\left(\sigma_{0}\right)=250 \mathrm{MPa}$ and the standard deviation $\sigma=0.1 E\left(\sigma_{0}\right)$.The reliability level is assumed $\psi=0.9999$. Let us calculate limit and shakedown load factors.

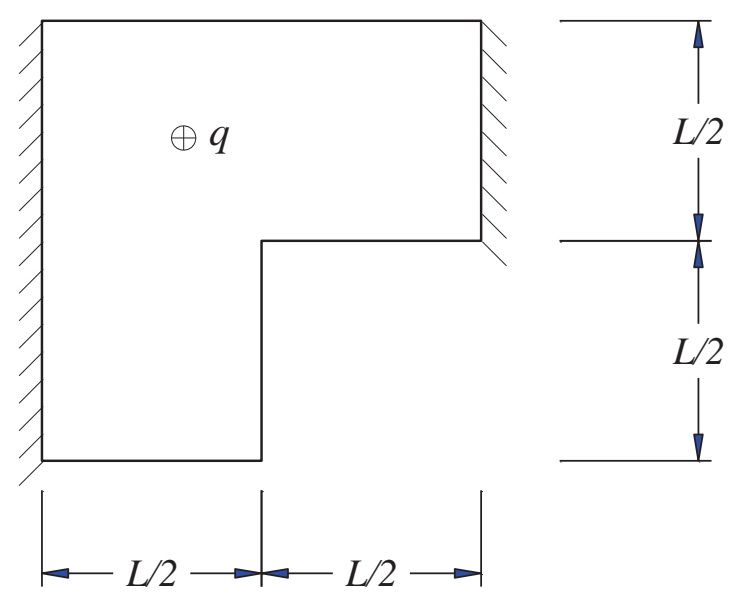

Figure 2: L-shape plate loaded by a uniform pressure 


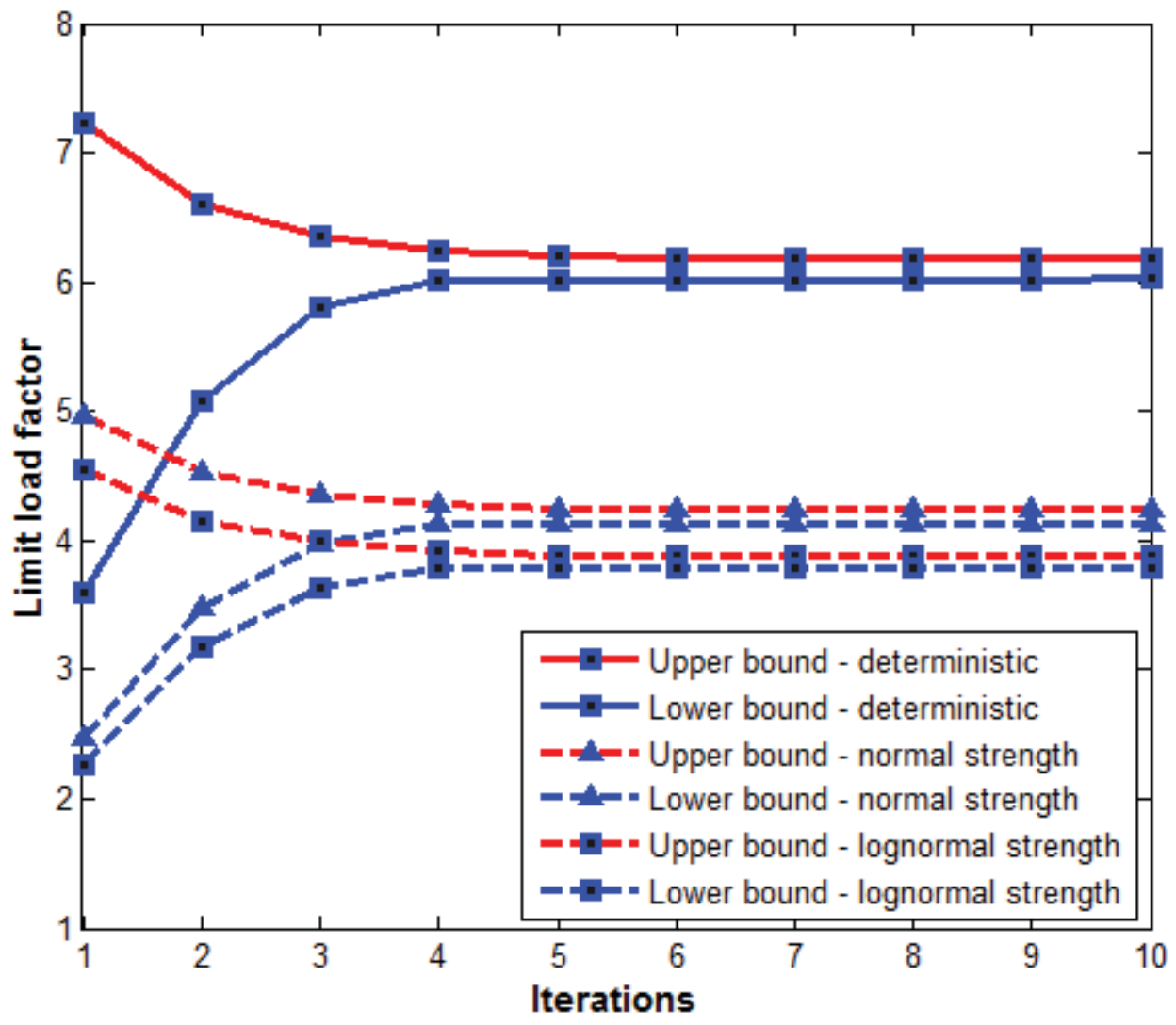

Figure 3: Convergence of limit load factors
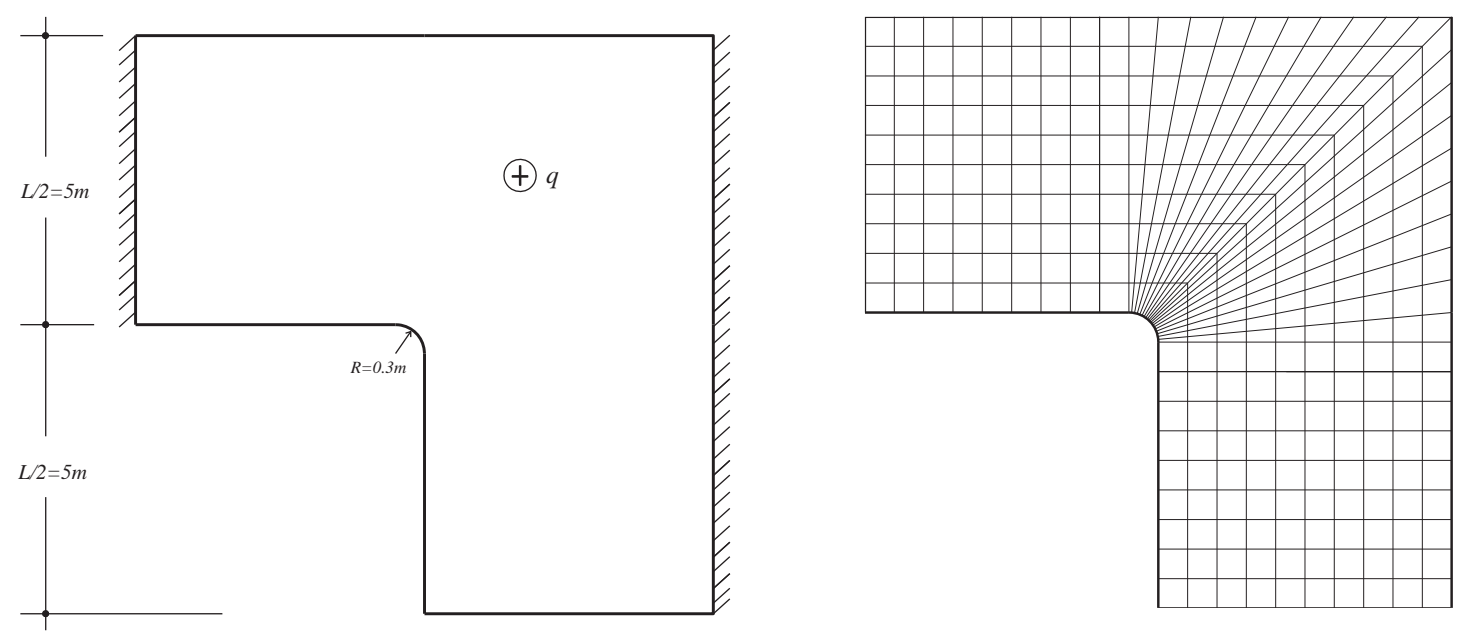

Figure 4. L-shape Plate: rounding at the corner 


\begin{tabular}{lccl}
\hline Authors & Lower bound & Upper bound & \\
\cline { 1 - 3 } Le et al. [15] & - & 6.219 & \\
\cline { 1 - 3 } Tran et al. [24] & 6.044 & 6.173 & \\
\hline \multirow{2}{*}{ Present } & 6.022 & 6.190 & \\
\cline { 2 - 4 } & 3.785 & 3.882 & normal \\
\cline { 2 - 4 } & 4.135 & 4.242 & lognormal \\
\hline
\end{tabular}

Table 1: Limit load factor in comparison for case of simple supported plate

For shakedown analysis, the stress singularity at the sharp reentrant corner has to be removed by rounding the plate at the corner as shown in Figure 4. The FE mesh is made with 380 DKQ elements. Table 2 shows the limit and shakedown load factors if a uniform load varies in the domain $q=\left[\begin{array}{l}0 \\ 1\end{array}\right]$. If we compare with the upper and lower bounds in Table 2, the limit load factors are similar. The convergence of shakedown load factors is shown in Figure 5.

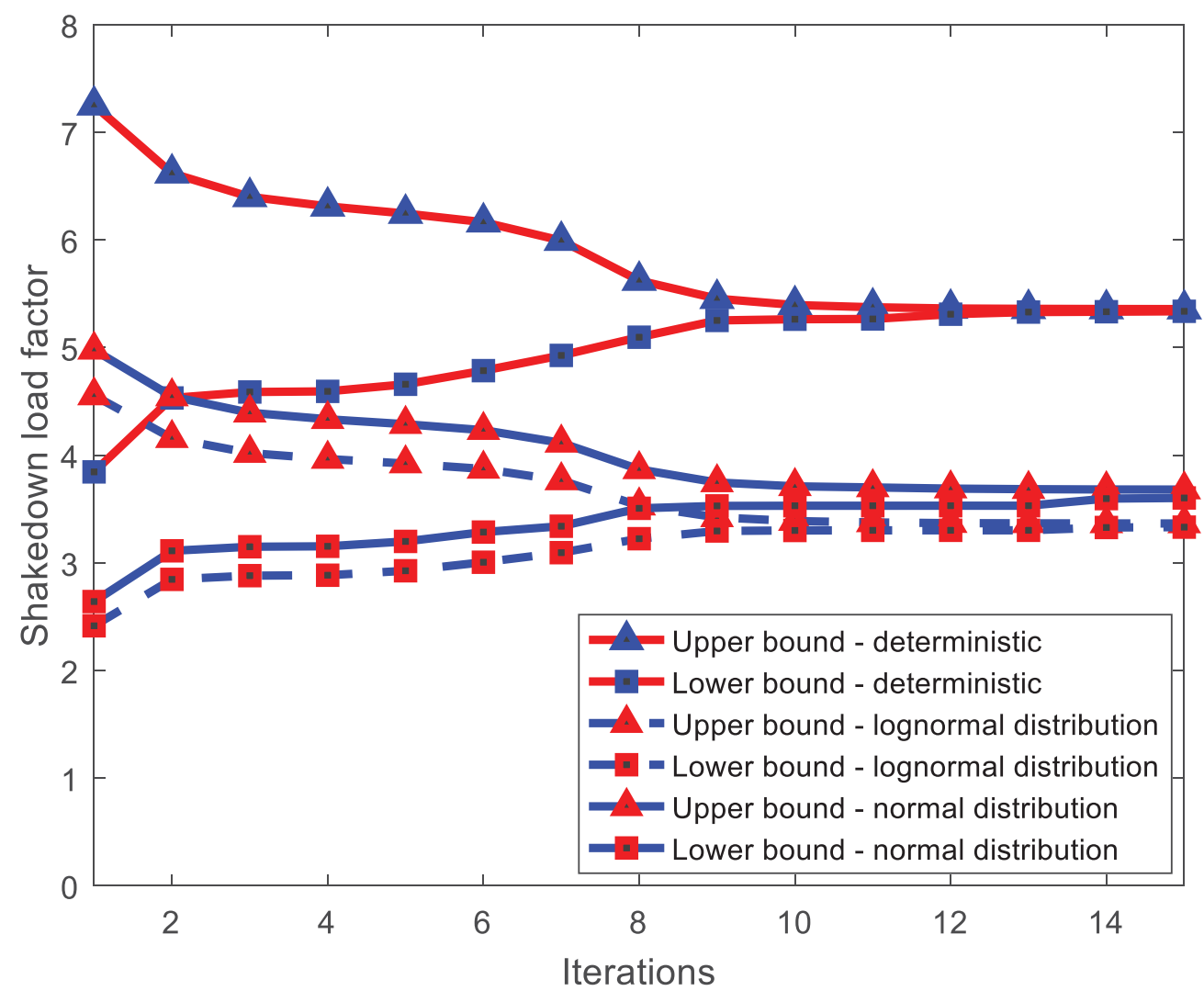

Figure 5: Convergence of Shakedown load factors 


\begin{tabular}{lccl}
\hline & Lower bound & Upper bound & \\
\hline \multirow{3}{*}{ Limit analysis } & 5.979 & 6.224 & deterministic \\
\cline { 2 - 4 } & 4.137 & 4.273 & lognormal \\
\cline { 2 - 4 } & 3.805 & 3.909 & normal \\
\hline \multirow{3}{*}{ Shakedown analysis } & 5.339 & 5.355 & deterministic \\
\cline { 2 - 4 } & 3.619 & 3.677 & lognormal \\
\cline { 2 - 4 } & 3.363 & 3.382 & normal \\
\hline
\end{tabular}

Table 2: Limit and shakedown load factors for plate in Figure 4

\section{CONCLUSIONS}

Reliability analysis of plates and shells calculates the failure probability after structural design for a given loading [13]. We have presented a probabilistic design method for plates, which allows the most effective numerical calculation of limit and shakedown loads for a prescribed failure probability of the structure with stochastic plastic moment. The implementation of the extension to stochastic loading, obtained in [2], is under preparation. The stochastic programming approach can be proposed as a method for structural optimization. Skakedown analysis has the advantage that it yields a design, which is optimum for all possible time-variant loadings in a considered load domain [41], [42].

\section{REFERENCES}

[1] G. Kirchhoff, Über das Gleichgewicht und die Bewegung einer elastischen Scheibe. $J$. reine angew. Math. 40: 51-88, 1850.

[2] N.T. Trần, M. Staat, Direct plastic structural design under random strength and random load by chance constrained programming. Eur J Mech A Solids, 85(1), art. no. 104106, 2021.

[3] N. T. Trần, M. Staat, Direct plastic structural design under lognormally distributed strength by chance constrained programming. Optim. Eng. 21(1), 131-157, 2020.

[4] Ngọc Trình Trần, Limit and Shakedown analysis of structures under stochastic conditions. PhD thesis, Technische Universität Carolo-Wilhelmina zu Braunschweig, Braunschweig, Germany, 2018.

[5] N.T. Trần, T.N. Trần, H.G. Matthies, G.E. Stavroulakis, M. Staat, Shakedown analysis under stochastic uncertainty by chance constrained programming. O. Barrera, A. Cocks, A. Ponter eds. Advances in direct methods for materials and structures. Springer, Cham, Heidelberg, 85-103, 2018.

[6] M. Staat, Limit and shakedown analysis under uncertainty. Int J Comput Methods, 11(3), Article ID 1343008, 2014.

[7] M. Staat, M. Heitzer, Probabilistic limit and shakedown problems. M. Staat, M. Heitzer eds. Numerical methods for limit and shakedown analysis. Deterministic and probabilistic approach. Part VII. NIC Series Vol. 15, John von Neumann Institute for Computing, Jülich, 217-268, 2003. http://hdl.handle.net/2128/2926 
[8] M.A. Save, C.E. Massonnet, G. de Saxcé, Plastic limit analysis of plates, shells and disks. $2^{\text {nd }}$ Edition, North Holland, 1997.

[9] D. Khoi Vu, Dual limit and shakedown analysis of structures. PhD thesis. Collection des publications de la Faculté des Sciences Appliquées, Université de Liège, Belgique, 2001 .

[10] N.T. Trần, T.N. Trần, H.G. Matthies, G.E. Stavroulakis, M. Staat, Shakedown analysis of plate bending under stochastic uncertainty by chance constrained programming. Proc. VII Eur. Congr. Comput. Methods Appl. Sci. Eng. (ECCOMAS Congr. 2016), no. June, pp. 3007-3019, 2016.

[11] N.T. Trần, T.N. Trần, H.G. Matthies, G.E. Stavroulakis, M. Staat, Shakedown analysis of plate bending analysis under stochastic uncertainty by chance constrained programming. M. Papadrakakis, V. Papadopoulos, G. Stefanou, V. Plevris eds. ECCOMAS Congress 2016, VII European Congress on Computational Methods in Applied Sciences and Engineering. Crete Island, Greece, 5-10 June 2016, Vol. 2, pp. 3007-3019, 2016.

[12] T.N. Trần, M. Staat, Shakedown analysis of Reissner-Mindlin plates using the edgebased smoothed finite element method. K. Spiliopoulos, D. Weichert eds. Limit states of materials and structures: Direct methods. Springer, Dordrecht, 101-117, 2014.

[13] T.N. Trần, R. Kreißig, M. Staat, Probabilistic limit and shakedown analysis of thin plates and shells. Structural Safety, 31(1), 1-18, 2009.

[14] C.V. Le, H. Nguyen-Xuan, H. Nguyen-Dang, Upper and lower bound limit analysis of plates using FEM and second-order cone programming. Comput. Struct., 88(1-2), 65-73, 2010.

[15] C.V Le, M. Gilbert, H. Askes, Limit analysis of plates using the EFG method and second-order cone programming. Int. J. Numer. Meth. Engng, 78, 1532-1552, 2009

[16] T. Belytschko, P.G. Hodge, Numerical methods for the limit analysis of plates. Trans. ASME, J. Appl. Mech., 35, 796-801, 1968.

[17] C.T. Morley, The ultimate bending strength of reinforced concrete slabs. PhD thesis, Cambridge University, 1965.

[18] L. Capsoni, A. Corradi, Limit analysis of plates-a finite element formulation. Struct. Eng. Mech., 8(4), 325-341, 1999.

[19] E.N. Fox, Limit analysis for plates: the exact solution for a clamped square plate of isotropic homogeneous material obeying the square yield criteron and loaded by uniform pressure. Math. Phys. Eng. Sci., 277(1265), 121-155, 1974.

[20] R.H. Wood, A partial failure of limit analysis for slabs, and the consequences for future research. Mag.Concr. Res., 21, 79-90, 1969.

[21] W.C. McCarthy, L.A. Traina, A plate bending finite element model with a limit analysis capacity. Math. Model., 8(Supplement C),486-492, 1987.

[22] K. Krabbenhoft, L. Damkilde, Lower bound limit analysis of slabs with nonlinear yield criteria. Comput. Struct., 80(27-30), 2043-2057, 2002.

[23] S. Timoshenko, S. Woinowsky-Krieger, Theory of plates and shells. 2nd Edition. McGraw Hill,1959. 
[24] T.N. Tran, A dual algorithm for shakedown analysis of plate bending. Numer. Methods Eng., 86(7), 862-875, 2011.

[25] J. Björnberg and M. Diehl, Approximate robust dynamic programming and robustly stable MPC. Automatica, 42(5), 777-782, 2006.

[26] L. Zéphyr, P. Lang, B. F. Lamond, P. Côté, Approximate stochastic dynamic programming for hydroelectric production planning. Eur. J. Oper. Res., 262(2), 586-601, 2017.

[27] K. Fukushima, Y. Waki, A polyhedral approximation approach to concave numerical dynamic programming. J. Econ. Dyn. Control, 37(11), 2322-2335, 2013.

[28] B. Srinivasan, S. Palanki, D. Bonvin, Dynamic optimization of batch processes: I. Characterization of the nominal solution. Comput. Chem. Eng., 27(1), 1-26, 2003.

[29] B. Srinivasan, D. Bonvin, E. Visser, S. Palanki, Dynamic optimization of batch processes: II. Role of measurements in handling uncertainty. Comput. Chem. Eng., 27(1), 27-44, 2003.

[30] G. Francois, D. Bonvin, Chapter One - Measurement-based real-time optimization of chemical processes. S. Pushpavanam ed. Control and Optimisation of Process Systems, vol. 43, Academic Press, 1-50, 2013.

[31] S. Rasoulian, L.A. Ricardez-Sandoval, Worst-case and distributional robustness analysis of a thin film deposition process. IFAC-PapersOnLine, 48(8), 1126-1131, 2015.

[32] Z.K. Nagy, R.D. Braatz, Distributional uncertainty analysis of a batch crystalization process using power series and polynomial chaos expansions. IFAC Proc. Vol., 39(2), 655-660, 2006.

[33] M. Skelin, M. Geilen, F. Catthoor, S. Hendseth, Worst-case performance analysis of SDF-based parameterized dataflow. Microprocess. Microsyst., 52, 439-460, 2017.

[34] Y. Aliari, A. Haghani, Planning for integration of wind power capacity in power generation using stochastic optimization. Renew. Sustain. Energy Rev., 59, 907-919, 2016.

[35] M. Riis, K.A. Andersen, Applying the minimax criterion in stochastic recourse programs. Eur. J. Oper. Res., 165(3), 569-584, 2005.

[36] S. Zier, K. Marti, Limit load analysis of plane frames under stochastic uncertainty. D. Weichert and A. Ponter eds. Limit states of materials and structures. Springer Netherlands, 113-134, 2009.

[37] A. Prepoka, Stochastic programming. Springer Netherlands, 1995.

[38] A. Charnes, W. Cooper, G.H. Symonds, Cost horizons and certainty equivalence: An approach in stochastic programming of heating oil. Manage. Sci., 4, 235-263, 1958.

[39] A. Charnes, W.W. Cooper, Chance-constrained programming. Manage. Sci., 6(1), 7379, 1959.

[40] T.N. Trần, R. Kreißig, Duc Khôi Vu, M. Staat: Upper bound limit and shakedown analysis of shells using the exact Ilyushin yield surface. Computers \& Structures, 86(1718),1683-1695, 2008. 
[41] K. Wiechmann, E. Stein, Shape optimization for elasto-plastic deformation under shakedown conditions. Int J Solids Struct, 43(22-23), 7145-7165, 2006.

[42] J. Atkočiūnas, L. Rimkus, V. Skaržauskas, E. Jarmolajeva, Optimal shakedown design of plates. Mechanika, 67(5), 14-23, 2007. 